\title{
Effects of SIRT1 silencing on viability, invasion and metastasis of human glioma cell lines
}

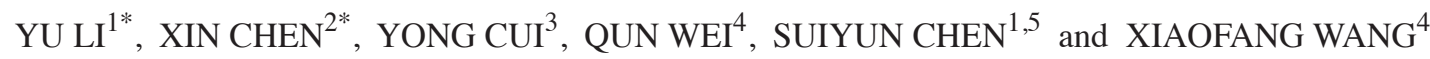 \\ ${ }^{1}$ School of Life Sciences, Yunnan University, Kunming, Yunnan 650091; ${ }^{2}$ Department of Orthopedics, \\ The Third People's Hospital of Yunnan Province, Kunming, Yunnan 650011; ${ }^{3}$ School of Agriculture and Biotechnology, \\ Yunnan Agricultural University, Kunming, Yunnan 650091; ${ }^{4}$ Department of Pathology, \\ The Second Affiliated Hospital of Kunming Medical University, Kunming, Yunnan 650101; \\ ${ }^{5}$ Biocontrol Engineering Research Center of Plant Disease \& Pest, Yunnan University, Kunming, Yunnan 650091, P.R. China
}

Received May 11, 2018; Accepted January 21, 2019

DOI: $10.3892 / \mathrm{ol} .2019 .10063$

\begin{abstract}
Silent information regulator 1 (SIRT1), a member of the sirtuin family, is involved in the development of various types of tumor. Previous studies have revealed that SIRT1 has dual functions, as a promoter and an inhibitor, in certain tumors. However, the role of SIRT1 in invasion and metastasis of glioma cells and its associated signaling pathway remain unclear. The aim of the present study was to determine the effects of SIRT1 on these processes and on the epithelial-mesenchymal transition (EMT) in human glioma and adjacent tissues, and in the human glioma cell lines U87 and U251. SIRT1 expression in tissues was investigated using the reverse transcription-quantitative polymerase chain reaction, western blotting and immunohistochemistry. The U87 and U251 cell lines were divided into control and SIRT1-small interfering RNA (siRNA) groups. The Cell Counting Kit-8, cell invasion assays were used to evaluate the effects of SIRT1 silencing on cell viability, invasion and EMT. Results indicated that SIRT1 was highly expressed in glioma tissues compared with in adjacent brain tissues. In addition, SIRT1-siRNA significantly inhibited the viability and invasion of U87 and U251 cells. Furthermore, EMT analysis revealed that the expression levels of the mesenchymal markers fibronectin and vimentin were significantly lower in the SIRT1-siRNA group compared with in the control group. Conversely,
\end{abstract}

Correspondence to: Dr Xiaofang Wang, Department of Pathology, The Second Affiliated Hospital of Kunming Medical University, 374 Dianmian Road, Kunming, Yunnan 650101, P.R. China

E-mail: wangxiaofangbio@126.com

Dr Suiyun Chen, School of Life Sciences, Yunnan University, 2 Cuihu North Road, Kunming, Yunnan 650091, P.R. China

E-mail: chensuiyun@ynu.edu.cn

*Contributed equally

Key words: silent information regulator 1, gliomas, viability, epithelial-mesenchymal transition expression levels of the epithelial markers epithelial cadherin and $\beta$-catenin were significantly higher in the SIRT1-siRNA group compared with in the control group. In conclusion, the results of the present study indicated that SIRT1 was positively associated with viability and invasion of U87 cells, potentially through EMT. These results suggested that SIRT1 may serve a crucial role in the proliferation and development of glioma.

\section{Introduction}

Glioma is one of the most common malignant tumors observed in China with a high degree of malignancy (1). Gliomas account for between 35 and $50 \%$ of all intracranial brain tumors in adult patients (2). Silent information regulator 1 (SIRT1) is a conserved class III deacetylase that deacetylates the lysine residues of nucleoproteins to influence their stability and transcriptional activities $(3,4)$. In addition, SIRT1 regulates cellular stress responses and lifespan (5). Despite great progresses in surgical treatment options for patients with gliomas, including chemotherapy and radiotherapy, the prognosis remains poor (6). Further investigation is therefore crucial to develop novel gene therapies for the treatment of glioma, and to elucidate the underlying molecular mechanisms of glioma development and progression.

SIRT1 is associated with cell viability and inhibition of apoptosis $(7,8)$, and is involved in the growth of certain tumors $(9,10)$. SIRT1 overexpression promotes tumor progression by regulating tumor growth-associated signaling pathways, including Wnt signaling $(11,12)$. A previous study reported that SIRT1 serves numerous roles in cancer biology (13). On the one hand, SIRT1 is upregulated in tumors, while cancer cells can downregulates the expression of tumor suppressor genes (14). On the other hand, SIRT1 can be proapoptotic (15) and anti-proliferative (16), and consequently has been proposed to behave as a tumor suppressor in vivo.

The epithelial-mesenchymal transition (EMT) is a biological process in which epithelial cells lose their polarity and adhesion properties, and differentiate into mesenchymal cells that possess migratory and invasive properties (17). EMT is a crucial biological process involved in the migration and invasion of certain tumor cells (18), and serves an important 
role in cancer progression and fibrosis (19). EMT is associated with tumorigenesis and metastasis. Previous studies identified that SIRT1 promotes prostate cancer growth and migration through EMT (20), and stimulates EMT and metastasis in colorectal cancer (21). Conversely, other studies revealed that SIRT1 suppresses EMT in cancer metastasis, organ fibrosis and nasal polypogenesis $(22,23)$. SIRT1 promotes the viability and inhibits apoptosis of human glioma cells (24). SIRT1 is also vital for neural stem cells maintenance and oncogenic transformation (25).

Numerous studies have identified various associations of SIRT1 with certain processes involved in cancer; however, its effects on invasion and EMT in human glioma remain unclear. Since controversies exist regarding the role of SIRT1 in tumors, as SIRT1 can downregulate the expression of tumor suppressor genes (14), SIRT1 also has been proposed to behave as a tumor suppressor in vivo (16), therefore, further investigation is required. To the best of our knowledge, the present study was the first to examine the effect of SIRT1 silencing on EMT in glioma. To do so, the expression levels of SIRT1 were analyzed in human glioma tissue samples together with the effects of SIRT1 on human glioma cell invasion. Previous studies reported that matrix metalloproteinase-9 (MMP-9) (26), Twist family basic helix-loop-helix transcription factor 1 (Twist1) and Snail family transcriptional repressor 1 (Snail1) serve important roles in tumor invasion (27). Therefore, these protein expression levels were also detected. The results indicated that SIRT1 was highly expressed in human glioma tissue samples compared with in adjacent tissues, and that SIRT1 silencing inhibited human glioma U87 and U251 cell line viability and invasion. In addition, SIRT1 silencing suppressed EMT in U87 and U251 cell lines, which suggested that SIRT1 may serve a role in EMT. In conclusion, the results of the present study provide an important foundation for further investigation of the underlying molecular mechanism of SIRT1 in glioma growth.

\section{Materials and methods}

Tissue specimen collection. A total of 20 glioma tissues and adjacent brain tissues were collected at The Second Affiliated Hospital of Kunming Medical University (Kunming, China) between April 2016 and April 2017. Tissues were collected following surgical resection. Tissue histomorphology was confirmed by pathologists. The present study was approved by the Ethics Committee of The Second Affiliated Hospital of Kunming Medical University and patients provided written informed consent.

Immunohistochemistry. Tissues are fixed in $4 \%$ paraformaldehyde for $24 \mathrm{~h}$ at room temperature. Fixed tissues were dehydrated with various concentrations of xylene and ethanol (50\% ethanol for $4 \mathrm{~h} ; 75 \%$ ethanol for $4 \mathrm{~h} ; 85 \%$ ethanol for $3 \mathrm{~h}$; $95 \%$ ethanol for $2 \mathrm{~h}$; $100 \%$ ethanol for $1 \mathrm{~h} ; 100 \%$ ethanol for $1 \mathrm{~h}$; 1:1 ethanol-xylene for $1 \mathrm{~h}$; xylene for $1 \mathrm{~h}$; xylene for $30 \mathrm{~min}$ at room temperature), embedded in paraffin. Sections ( $4 \mu \mathrm{m}$ thickness) were cut from a paraffin block. Sections were dewaxed with various concentrations of xylene and ethanol (xylene for $10 \mathrm{~min}$; xylene for $5 \mathrm{~min}$; $100 \%$ ethanol for $5 \mathrm{~min}$; $95 \%$ ethanol for $2 \mathrm{~min}$; $80 \%$ ethanol for $2 \mathrm{~min} ; 70 \%$ ethanol for $2 \mathrm{~min}$ ). Antigen repair was performed on the sections with $0.01 \mathrm{M}$ citric acid buffer ( $\mathrm{pH} 6.0$ ) at $100^{\circ} \mathrm{C}$ high temperature and $80 \mathrm{kpa}$ pressure. Sections were blocked by incubation with $5 \%$ goat serum (Beijing Solarbio Science \& Technology Co., Ltd., Beijing, China) in PBS for $15 \mathrm{~min}$ at room temperature. Sections were incubated with anti-SIRT1 rabbit antibody (1:100; cat. no. 13161-1-AP; ProteinTech Group, Inc., Chicago, IL, USA) overnight at $4^{\circ} \mathrm{C}$ and with a HRP Goat Anti-Rabbit IgG antibody (1:200; cat. no. AS014, ABclonal Biotech Co., Ltd., Wuhan, China) for $2 \mathrm{~h}$ at room temperature. The reactions were visualized using a 3,3'-diaminobenzidine visualization kit (Fuzhou Maixin Biotech Co., Ltd., Fuzhou, China). Sections were counterstained with hematoxylin to visualize nuclei, for 5-10 $\mathrm{min}$ at room temperature. Sections were examined under a light microscope, (x400, magnification). Brown staining indicated immunoreactive positive cells, and blue staining indicated the nuclei.

Cell culture. The human glioblastoma cell line U251 and the glioblastoma cell line U87 of unknown origin were purchased from The Kunming Cell Bank of the Chinese Academy of Sciences (Kunming, China). Cells were cultured in Dulbecco's modified Eagle's medium (DMEM) supplemented with $10 \%$ fetal bovine serum (FBS) and antibiotics (Gibco; Thermo Fisher Scientific, Inc., Waltham, MA, USA) and placed at $37^{\circ} \mathrm{C}$ in a humidified incubator containing $5 \% \mathrm{CO}_{2}$.

SIRT1 silencing. SIRT1-small interfering RNA (siRNA) was used to target the SIRT1 gene. The nucleotide sequences were synthesized from Sangon Biotech Co., Ltd., (Shanghai, China) as follows: Forward, 5'-ACUUUGCUGUAACCCUGU A-3' and reverse, 3'-UACAGGGUUACAGCAAAGU-3' (28). For comparison, a random nucleotide sequence (5'-CUAGCU UAUGUGGACCUCG-3') was used as a negative control. The transfections were carried out with Lipofectamine ${ }^{\circledR} 2000$ reagent (Invitrogen; Thermo Fisher Scientific, Inc.) according to the manufacturer's protocol. Cells were harvested at $48 \mathrm{~h}$ post-transfection, and mRNA and protein levels were analyzed using the reverse transcription-quantitative polymerase chain reaction (RT-qPCR) and western blotting, respectively.

Cell viability assay. Cell viability was evaluated using the Cell Counting Kit-8 (CCK-8) (Beyotime Institute of Biotechnology, Haimen, China). Cells $\left(1 \times 10^{4}\right)$ were seeded in a 96-well plate. At $48 \mathrm{~h}$ after transfection, cell viability was assessed using the CCK-8 assay, according to the manufacturer's protocol. Briefly, cells were incubated at $37^{\circ} \mathrm{C}$ for $1 \mathrm{~h}$, prior to measuring the optical density (OD) at $450 \mathrm{~nm}$ using a multiplate reader.

Cell invasion assays. A cell invasion assay was performed using $8 \mu \mathrm{m}$ Transwell chambers (BD Biosciences, San Jose, CA, USA) precoated with Matrigel. Briefly, $300 \mu 1$ cell suspension $\left(5 \times 10^{5}\right.$ cells $\left./ \mathrm{ml}\right)$ was added to the upper chamber, and $500 \mu \mathrm{l}$ DMEM containing 10\% FBS was added to the lower chamber. Following $48 \mathrm{~h}$ transfection, cells that did not invade the Matrigel were discarded. Filters were fixed in $90 \%$ ethanol for $10 \mathrm{~min}$ at room temperature, stained with $0.1 \%$ crystal violet for $5 \mathrm{~min}$ at room temperature, and visualized under a light microscope (magnification, $\mathrm{x} 100$ ). 
$R T$ - $q P C R$. Total RNA was isolated from cells and tissues using TRIzol ${ }^{\circledR}$ (Invitrogen; Thermo Fisher Scientific, Inc.). cDNA was generated using a Reverse Transcription (RT) kit (Vazyme Biotech Co., Ltd, Nanjing, China), according to the manufacturer's protocol. qPCR was performed using a SYBR qPCR Master Mix kit (Vazyme Biotech Co., Ltd.) in an ABI 7300 real-time PCR machine (Thermo Fisher Scientific, Inc.) according to the manufacturer's protocols. qPCRs were performed as follows: $95^{\circ} \mathrm{C}$ for $5 \mathrm{~min}$, followed by 40 cycles of $95^{\circ} \mathrm{C}$ for $15 \mathrm{sec}$ and $60^{\circ} \mathrm{C}$ for $30 \mathrm{sec}$. The relative expression of each mRNA of interest were normalized to endogenous control and analyzed using the $2^{-\Delta \Delta \mathrm{Cq}}$ method $(29,30)$. Epithelial (E-) cadherin, $\beta$-catenin, fibronectin, vimentin and $\beta$-actin primers were as follows: E-cadherin, 5'-ATGCTGAGGATGATTGAG GTGGGT-3' (forward) and 5'-CAAATGTGTTCAGCTCAG CCAGCA-3' (reverse); $\beta$-catenin, 5'-TGCAGTTCGCCTTCA CTATGGACT-3' (forward) and 5'-GATTTGCGGGACAAA GGGCAAGAT-3' (reverse); fibronectin, 5'-AAACTTGCA TCTGGAGGCAAACCC-3' (forward) and 5'-AGCTCTGAT CAGCATGGACCACTT-3' (reverse); vimentin, 5'-AGAACC TGCAGGAGGCAGAAGAAT-3' (forward) and 5'-TTCCAT TTCACGCATCTGGCGTTC-3' (reverse); and $\beta$-actin, 5'-TGA CGTGGACATCCGCAAAG-3' (forward) and 5'-CTGGAA GGTGGACAGCGAGG-3' (reverse).

Western blotting. At $48 \mathrm{~h}$ after transfection, total proteins from U87 and U251 cells were extracted using RIPA lysis buffer (Beyotime Institute of Biotechnology). Protein were quantified by using a bicinchoninic protein assay kit (Beyotime Institute of Biotechnology). Proteins $(30 \mu \mathrm{g})$ were separated by SDS-PAGE (10\% gel) and transferred onto a polyvinylidene fluoride membrane, which was blocked with $10 \%$ skimmed milk in Tris-buffered saline, $\mathrm{pH} 7.0$, containing $0.1 \%$ Tween-20 for $2 \mathrm{~h}$. Following blocking, membranes were incubated with primary antibodies at $4^{\circ} \mathrm{C}$ overnight (ProteinTech Group, Inc.) and followed by HRP Goat Anti-Rabbit IgG secondary antibodies (dilution 1:1,000; cat. no. AS014; ABclonal, Wuhan, China) for $2 \mathrm{~h}$ at room temperature. The primary antibodies as follows: SIRT1 antibody (dilution, 1:1,000; cat. no. 13161-1-AP; ProteinTech), MMP-9 antibody (dilution, 1:1,000; cat. no. 10375-2-AP; ProteinTech), Twist1 antibody (dilution, 1:1,000; cat. no. 25465-1-AP; ProteinTech), MMP-9 antibody (dilution, 1:1,000; cat. no. 10375-2-AP; ProteinTech), Snail1 antibody (dilution, 1:1,000; cat. no. 13099-1-AP; ProteinTech), E-cadherin antibody (dilution, 1:1,000; cat. no. 20874-1-AP; ProteinTech), $\beta$-catenin antibody (dilution, 1:1,000; cat. no. 51067-2-AP; ProteinTech), Fibronectin antibody (dilution, 1:1,000; cat. no. 15613-1-AP; ProteinTech), Vimentin antibody (dilution, 1:1,000; cat. no. 10366-1-AP; ProteinTech). Membrane signals were visualized with enhanced chemiluminescence reagent (Beyotime Institute of Biotechnology). The OD of the bands was determined using ImageJ 2x software (National Institutes of Health, Bethesda, MD, USA) and normalized to the expression of the internal control $(\beta$-actin).

Immunocytochemistry. Cells were fixed in $4 \%$ paraformaldehyde for $30 \mathrm{~min}$ at room temperature. Following three washes with $1 \mathrm{X}$ PBS, cells were blocked with normal goat serum for $2 \mathrm{~h}$. The cells were then incubated overnight at $4^{\circ} \mathrm{C}$ with anti-E-cadherin $(1: 100 ; 20874-1-A P$; ProteinTech Group, Inc.) or anti-fibronectin antibodies (dilution, 1:100; cat. no. 15613-1-AP; ProteinTech Group, Inc.) and with fluorescein isothiocyanate-conjugated secondary antibody (dilution, 1:100; cat. no. SA00003-2; ProteinTech Group, Inc.) or CoraLite594-conjugated secondary antibody (dilution, 1:100; cat. no. SA00013-4; ProteinTech Group, Inc.). Nuclei were stained with $10 \mu \mathrm{g} / \mathrm{ml}$ DAPI in the dark for $5 \mathrm{~min}$ at room temperature. Cells were observed using a fluorescence microscope (magnification, $x 100$ ). Three representative fields of stained cells were analyzed using Image-Pro Plus software (version 6.0; Media Cybernetics, Inc., Rockville, MD, USA) to obtain the mean OD, which represents the staining strength per positive pixel.

Statistical analysis. Differences between two groups were analyzed using Student's t-tests with GraphPad Prism software (version 5.0a; GraphPad Software, Inc., La Jolla, CA, USA). Data are presented as the mean \pm standard deviation $(n=3) . P<0.05$ was considered to indicate a statistically significant difference.

\section{Results}

SIRT1 expression in glioma tissues. SIRT1 expression was detected in 20 glioblastoma tissues and their corresponding adjacent non-tumor tissues using RT-qPCR, western blotting and immunohistochemistry. The results indicated that SIRT1 mRNA and protein expression levels were upregulated in tumor tissues compared with in adjacent non-tumor tissues (Fig. 1A and B), which was determined to be significant. Results from immunohistochemistry indicated that SIRT1 was present in the nucleus of glioma tissues (Fig. 1C). SIRT1 levels were different in glioma tissues compared with adjacent tissues. These results indicated that SIRT1 expression was associated with glioma development.

SIRT1 silencing inhibits glioma cell viability. To investigate the effects of SIRT1 on U87 and U251 cell viability, cells were transfected with SIRT1-siRNA. RT-qPCR and western blotting confirmed the successful SIRT1 silencing in U87 and U251 cell lines (Fig. 2A and B). A CCK-8 assay was used to detect the cell viability of U87 and U251 cells at $48 \mathrm{~h}$ after transfection with SIRT1-siRNA. Results indicated that U87 and U251 cell viabilities were significantly decreased following SIRT1-siRNA transfection compared with control transfection (Fig. 2C and D). These data suggested that SIRT1 expression may be involved in U87 and U251 cell viability.

Silencing SIRT1 inhibits invasion of glioma cells. To determine the effect of SIRT1 on cell invasion, Transwell invasion assays were performed on U87 and U251 cells transfected with SIRT1-siRNA. Results indicated that SIRT1-siRNA suppressed U87 and U251 cell compared with cells transfected with control siRNA (Fig. 3A). Images of cells present on the lower membranes of the invasion assay chambers and quantification of crystal violet-positive cells are presented in Fig. 3A, which indicated a significant difference for the two cell lines. Results indicated that SIRT1-siRNA inhibited U87 and U251 cell invasion. The expression levels of MMP-9, Twist1 and Snaill were therefore determined in U87 and U251 cell lines 
A

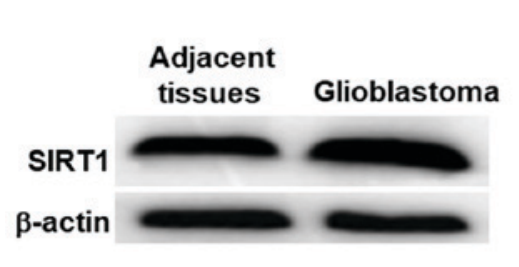

SIRT1

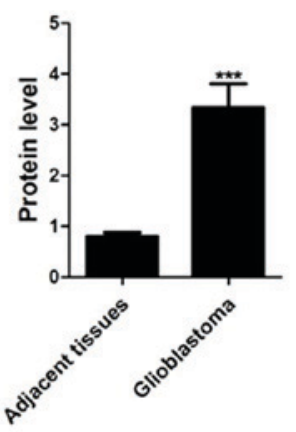

B

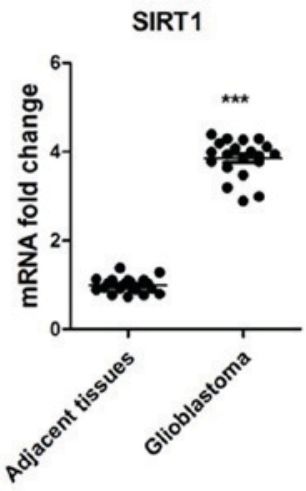

C
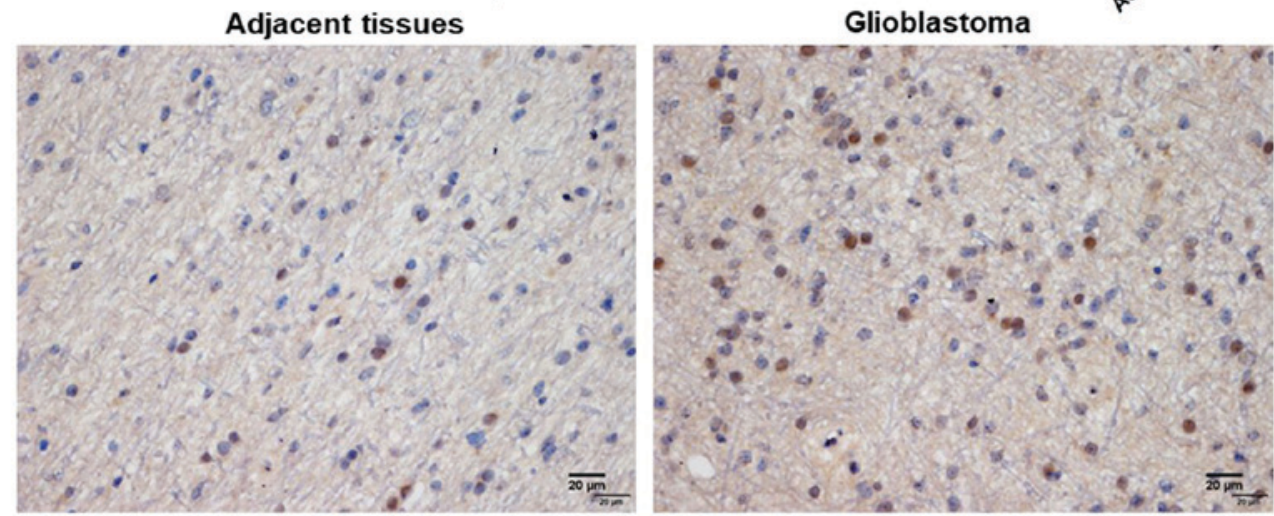

Figure 1. SIRT1 expression in glioma tissues and adjacent brain tissues. SIRT1 (A) protein expression, and (B) mRNA levels in glioma and adjacent brain tissues determined by western blotting and reverse transcription-quantitative polymerase chain reaction. (C) SIRT1 immunohistochemistry of glioma tissues and adjacent tissues (magnification $\mathrm{x} 400$ ). Results are presented as the mean \pm standard deviation of three independent experiments. ${ }^{* * *} \mathrm{P}<0.001$. SIRT1, silent information regulator 1 .

A
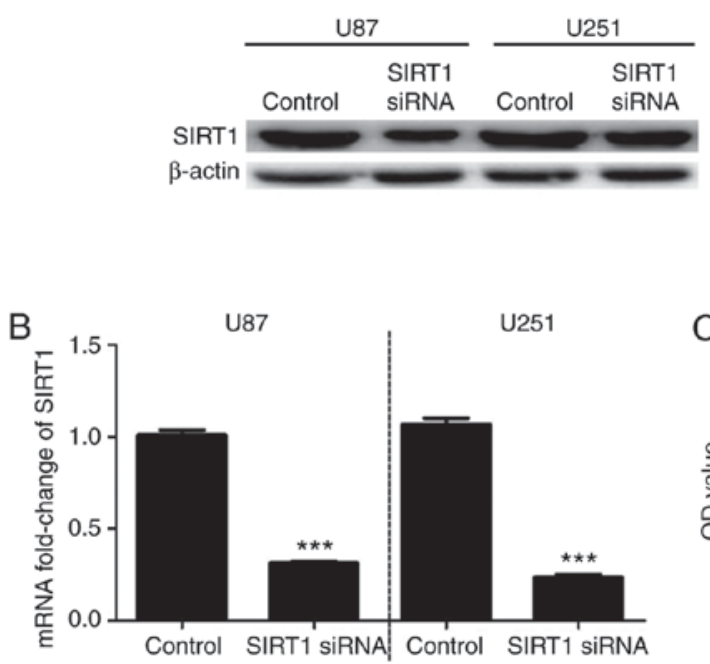
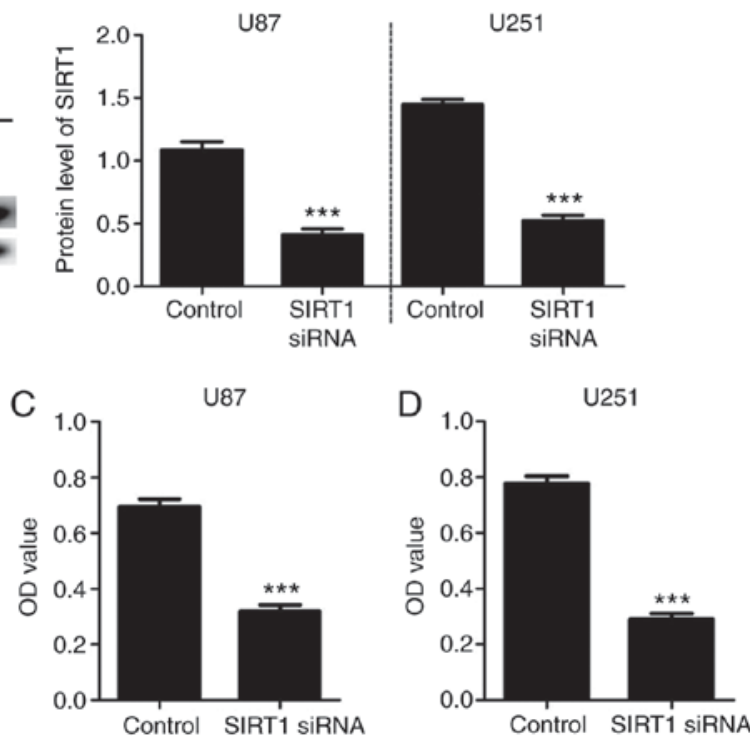

Figure 2. SIRT1 silencing inhibits glioma cell viability. SIRT1 (A) protein and (B) mRNA expression levels in U87 and U251 cells following transfection with SIRT1-siRNA. Cell viability of (C) U87 and (D) U251 cells following SIRT1-siRNA transfection. Results are presented as the mean \pm standard deviation of three independent experiments. ${ }^{* * *} \mathrm{P}<0.001$. vs. control. OD, optical density; siRNA, small interfering RNA; SIRT1, silent information regulator 1.

by western blotting. Results indicated that SIRT1-siRNA decreased the protein levels of MMP-9, Twist1 and Snail1 in the two cell lines (Fig. 3B and C), which suggested that SIRT1 may have a beneficial effect on glioma cell invasion.

SIRT1 silencing inactivates EMT pathway in glioma cells. The aforementioned results suggested that SIRT1 may promote glioma cell viability invasion. EMT is a crucial biological process for the invasion of certain types of malignant tumor cells. To identify the effect of SIRT1 on EMT in U87 and U251 cells, RT-qPCR and western blotting were used to detect the expression levels of the epithelial markers E-cadherin and $\beta$-catenin, and the mesenchymal markers fibronectin and vimentin. Results indicated that SIRT1 
A

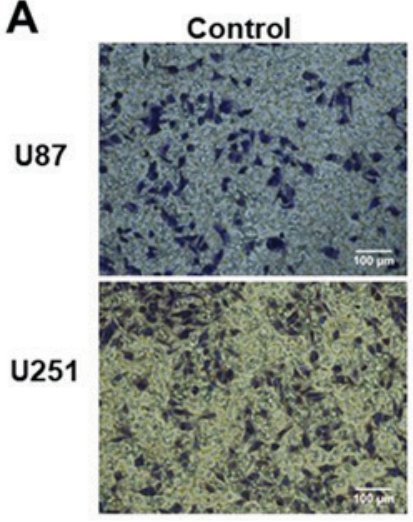

U87

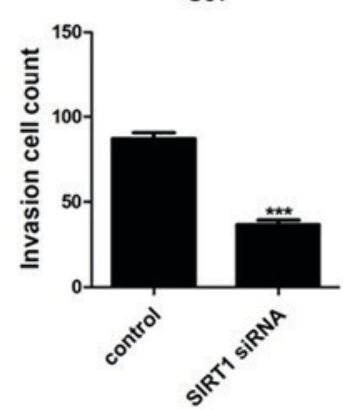

SIRT1 SIRNA

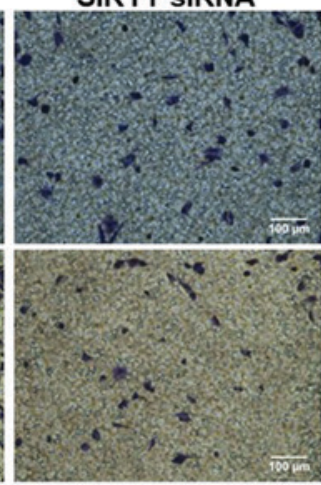

U251

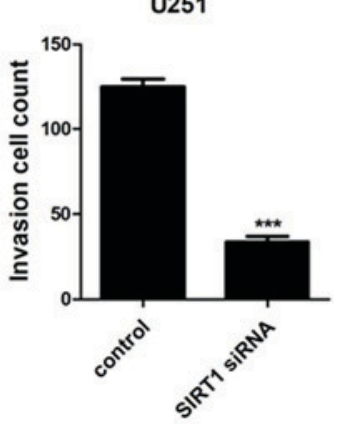

B

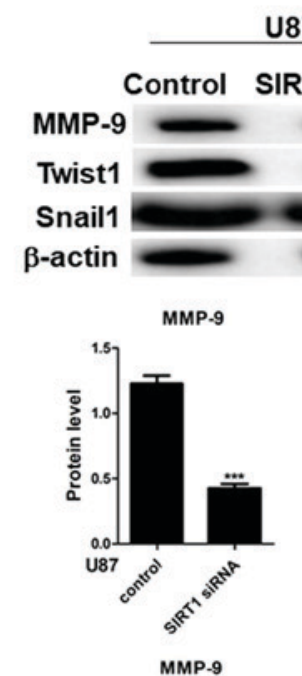

U87

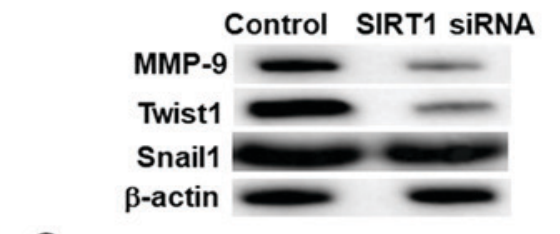

C
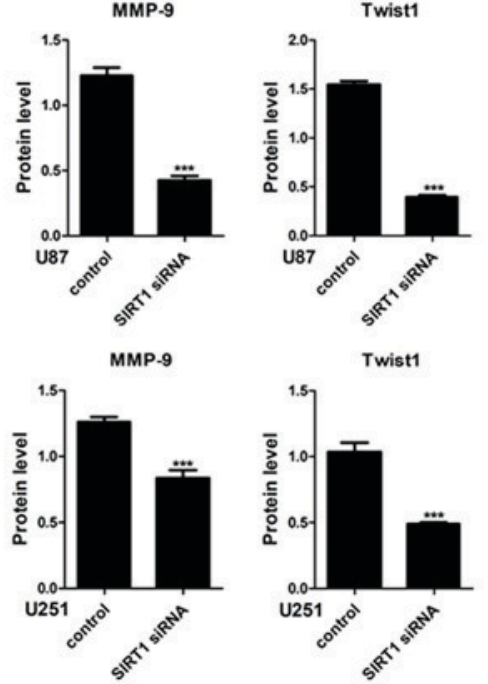

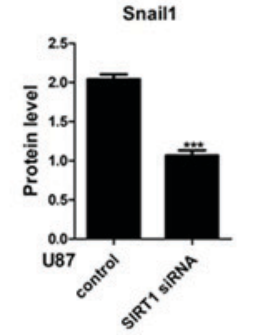

U251

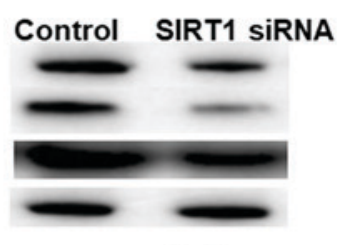

Snail1

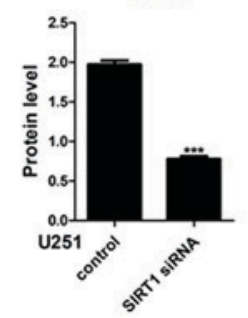

Figure 3. SIRT1 silencing inhibits invasion of glioma cells. (A) Invasive ability of U87 and U251 cells following transfection with SIRT1-siRNA determined using Transwell assays (magnification x100). (B) Western blotting of MMP-9, Twist1 and Snail1 in U87 and U251 cells following transfection with SIRT1-siRNA. (C) Quantification of protein levels. Results are presented as the mean \pm standard deviation of three independent experiments. ${ }^{* * *} \mathrm{P}<0.001$. MMP-9, matrix metalloproteinase-9; siRNA, small interfering RNA; SIRT1, silent information regulator 1, Snail1, snail family transcriptional repressor 1; Twist1, family basic helix-loop-helix transcription factor 1.

A

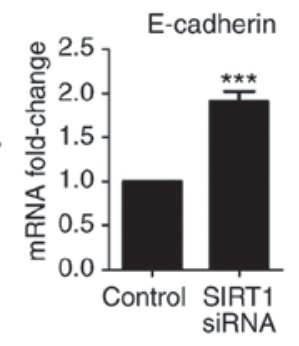

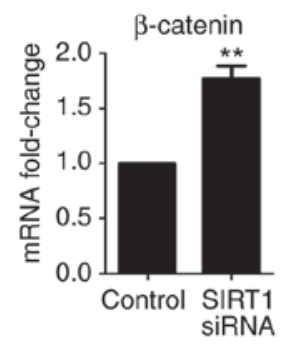
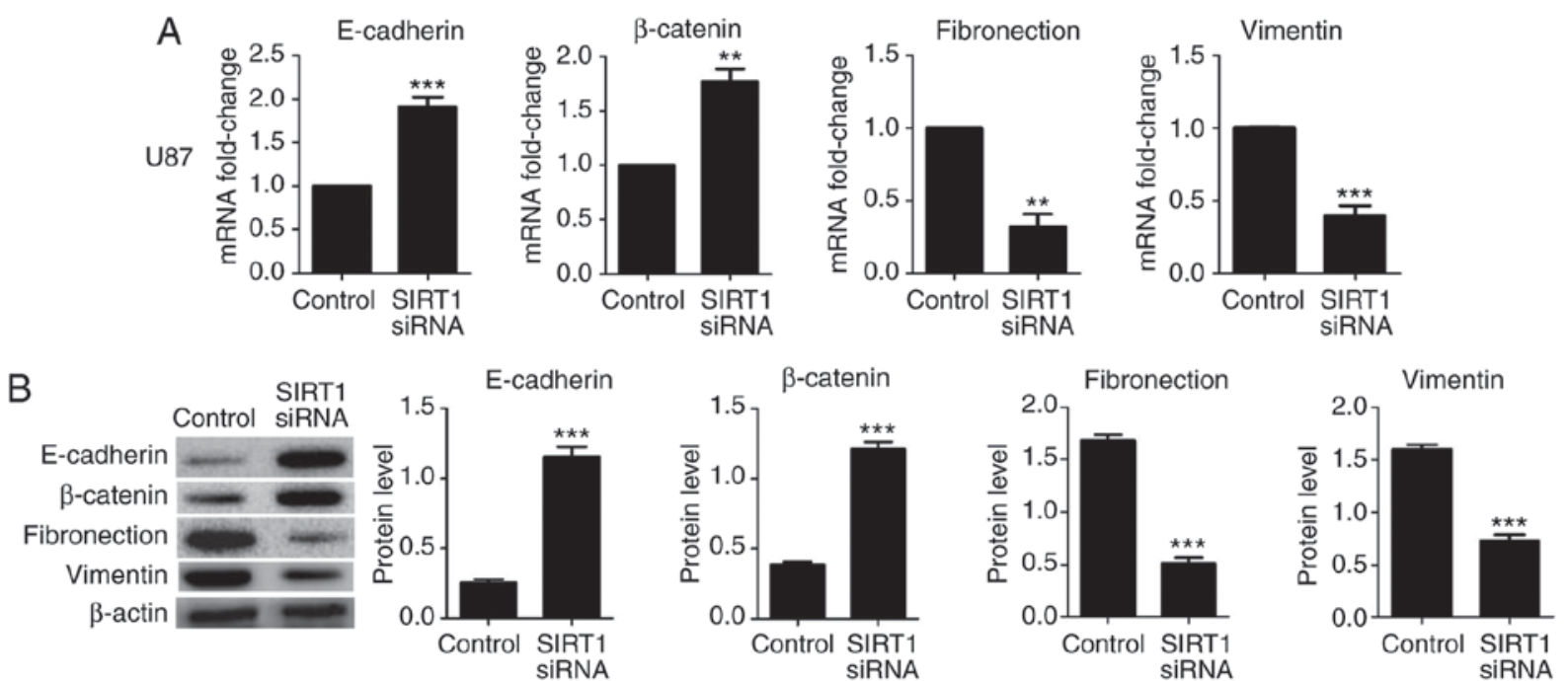

Figure 4. SIRT1 silencing inhibits epithelial-mesenchymal transition in U87 cells. The (A) mRNA and (B) protein levels of E-cadherin, $\beta$-catenin, fibronectin and vimentin in U87 cells following transfection with SIRT1-siRNA were determined using the reverse transcription-quantitative polymerase chain reaction and western blotting, respectively. "** $\mathrm{P}<0.01$ and ${ }^{* * * *} \mathrm{P}<0.001$. siRNA, small interfering RNA; SIRT1, silent information regulator 1; E-cadherin, epithelial cadherin.

silencing significantly increased the mRNA and protein levels of E-cadherin and $\beta$-catenin in U87 and U251 cells; conversely, SIRT1-siRNA significantly decreased the mRNA and protein levels of fibronectin and vimentin in U87 and U251 cells (Figs. 4 and 5, respectively). In addition, immunofluorescence analysis for E-cadherin and fibronectin revealed a similar significant fluorescence decrease following SIRT1 silencing (Fig. 6). These results indicate that SIRT1 may promote EMT in glioma cells.

\section{Discussion}

Glioma is one of the most common malignancies in China. In addition, it is the most frequent type of intracranial tumor and the 
A

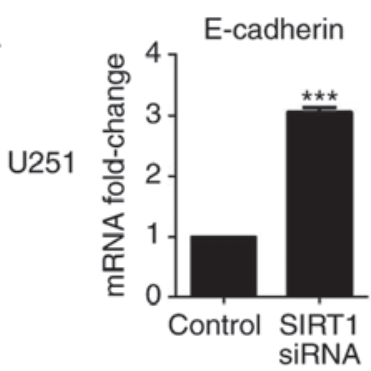

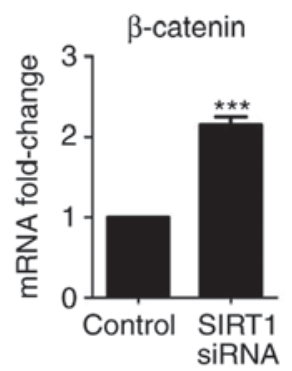
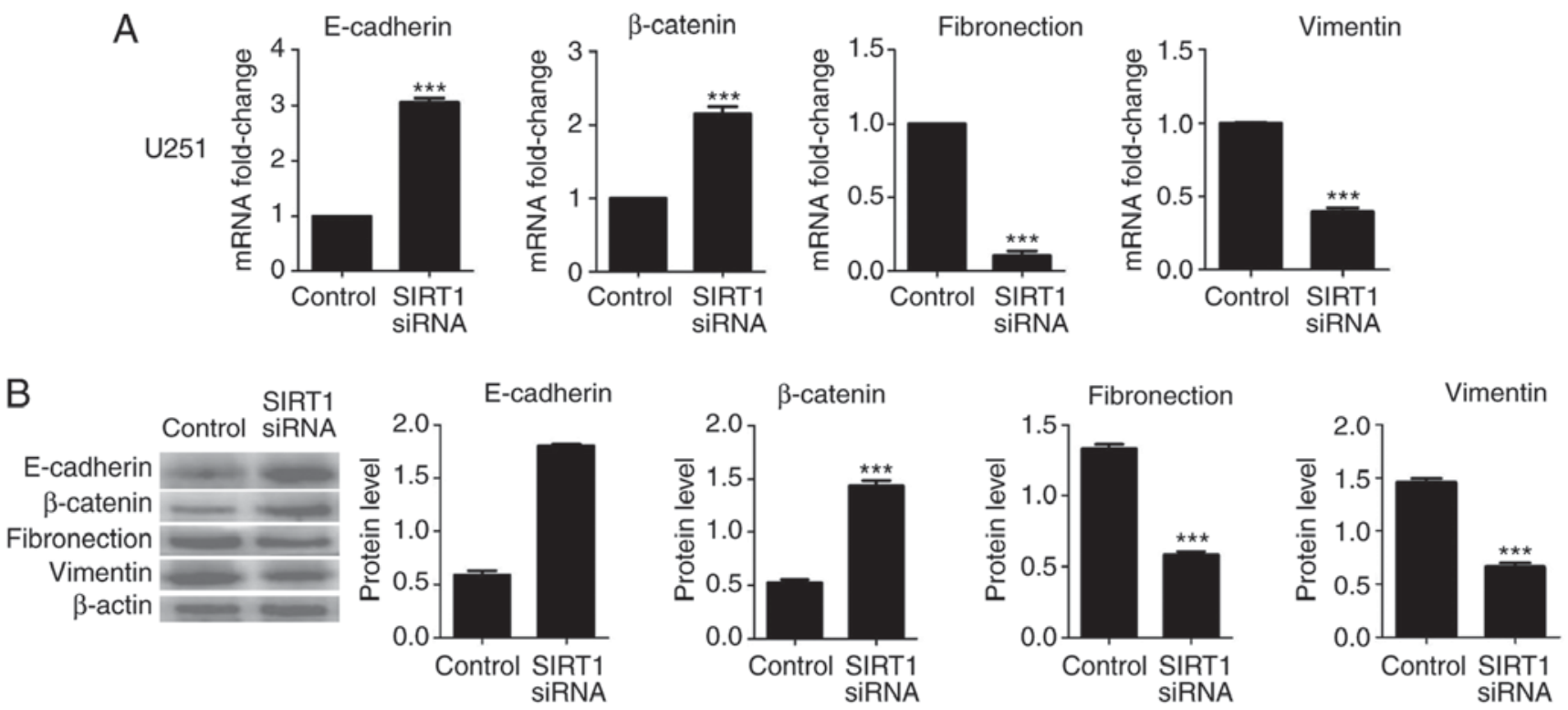

Figure 5. SIRT1 silencing inhibits epithelial-mesenchymal transition in U251 cells. The (A) mRNA and (B) protein levels of E-cadherin, $\beta$-catenin, fibronectin and vimentin in U251 cells following transfection with SIRT1-siRNA were determined using the reverse transcription-quantitative polymerase chain reaction and western blotting, respectively. ${ }^{* * *} \mathrm{P}<0.001$. siRNA, silencing RNA; SIRT1, silent information regulator 1; E-cadherin, epithelial cadherin.

A
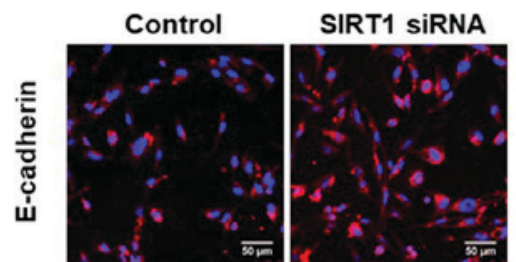

C
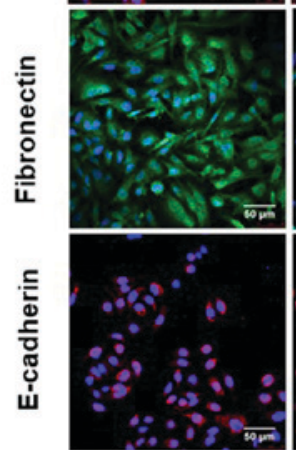

U251

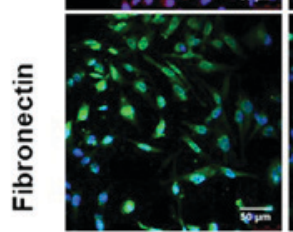

B

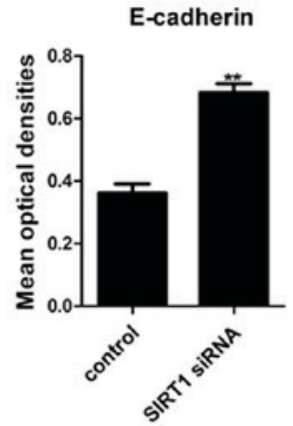

D

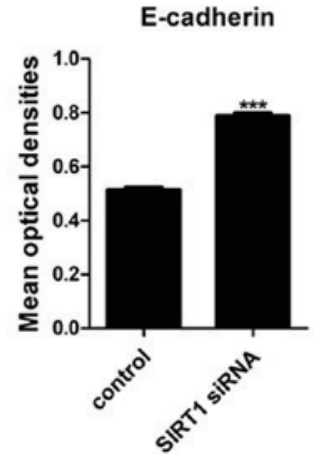

Fibronectin

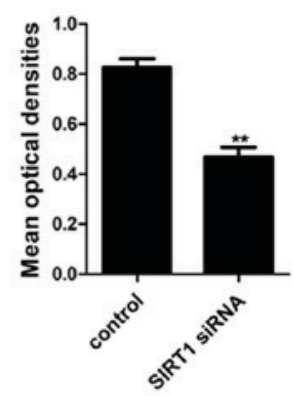

Fibronectin

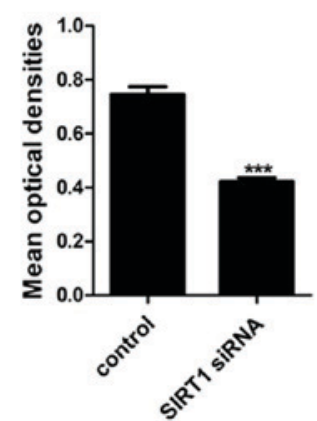

Figure 6. Immunofluorescence detection of E-cadherin and fibronectin. (A) Immunofluorescence and (B) quantification of results of E-cadherin and fibronectin in U87, following transfection with SIRT1-siRNA (magnification, x100). (C) Immunofluorescence and (D) quantification of results of E-cadherin and fibronectin in U251 cells following transfection with SIRT1-siRNA (magnification x100). Results are presented as the mean \pm standard deviation of three independent experiments. ${ }^{* *} \mathrm{P}<0.01$ and ${ }^{* * * *} \mathrm{P}<0.001$. siRNA, silencing RNA; SIRT1, silent information regulator 1; E-cadherin, epithelial cadherin.

most primary malignant brain tumor $(31,32)$. The World Health Organization classifies glioma according to its malignancy with grades ranging from I to IV (33). Although microsurgical tumor resection, adjuvant chemotherapy and radiotherapy are available, $75 \%$ of patients diagnosed with glioma succumb within 18 months of diagnosis (34). Further investigation aiming to develop novel therapeutic targets to prevent glioma metastasis is therefore crucial. To the best of our knowledge, the effect of SIRT1 on glioma remains poorly documented and the role of SIRT1 in EMT in glioma remains unclear.

SIRT1 is a class III histone deacetylase that belongs to the sirtuin family. The sirtuins have been reported to serve crucial roles in genome stability, stress responses and tumorigenesis (35). However, they serve a dual role in cancer. SIRT1 
is a bifunctional sirtuin that is involved in tumor suppression and other oncogenic factors (36); however, the mechanisms underlying these contradictory functions remain unclear. SIRT1 is upregulated in lung adenocarcinoma, colorectal, hepatocellular and prostate cancer $(10,36,37)$. SIRT1 inhibition can suppress tumor growth (38). In addition, the tumor suppressor p53 downregulates SIRT1 expression in glioma tumor cells (39). SIRT1 also induces p53 inactivation and inhibits p53-dependent apoptosis (7). Furthermore, SIRT1 promotes viability and inhibits apoptosis of glioma tumor cell lines (24). However, the effects of SIRT1 on invasion and metastasis and its underlying molecular mechanisms in glioma remain unknown. In the present study, the expression levels of SIRT1 in glioma tissues and adjacent brain tissue were determined using RT-qPCR, western blotting and immunohistochemistry. Results revealed that SIRT1 was overexpressed in glioma tissues, compared with adjacent brain tissues that only exhibited weak signal. In addition, SIRT1 silencing was used to investigate SIRT1 effects on invasion and metastasis of glioma U87 cells.

EMT is an important process involved in tumor metastasis $(40,41)$. Previous studies have identified that increased migration and invasion are positively associated with EMT $(42,43)$. In addition, decreased expression of E-cadherin and $\beta$-catenin, and increased expression of fibronectin and vimentin serve crucial roles in tumor progression (44). Furthermore, it was reported previously that EMT serves a crucial role in glioma $(45,46)$ and that SIRT1 induces EMT in various types of tumor (47). However, it was identified previously that SIRT1 suppresses EMT in cancer metastasis (22). In the present study, SIRT1 silencing suppressed viability and invasion of glioma U87 and U251 cells, increased E-cadherin and $\beta$-catenin expression, and decreased fibronectin and vimentin expression.

To the best of our knowledge, the present study was the first to assess the mRNA and protein expression levels of SIRT1 in human glioma and adjacent brain tissues. To do so, the effects of SIRT1 on viability and invasion of glioma U87 and U251 cells were investigated. Results indicated that SIRT1 expression was higher in glioma tissues compared with in adjacent brain tissues, and that SIRT1 silencing inhibited cell viability, invasion and metastasis in glioma U87 and U251 cells. Furthermore, SIRT1 silencing significantly decreased EMT in U87 and U251 cells, which suggested that EMT may contribute to the inhibition of viability, invasion and metastasis of glioma cells.

The preliminary results of the present study highlight the potential association of SIRT1 with EMT during human glioma progression; however, the underlying molecular mechanisms remain unclear and further investigation is required. Future work will investigate how SIRT1 influences the EMT associated pathway in human glioma.

In conclusion, the results of the present study may serve to further understand SIRT1 functions and mechanisms in glioma cells. These results suggested that SIRT1 may be considered as a potential novel therapeutic target for glioma.

\section{Acknowledgements}

Not applicable.

\section{Funding}

No funding was received.

\section{Availability of data and materials}

All data generated or analyzed during this study are included in this published article and are freely available to any researchers.

\section{Authors' contributions}

YL, XC and YC guided the experiments; XW and SC designed the experiments; XC, YC, QW and YL performed the experiments and analyzed the data; and YL wrote the manuscript. All authors have read and approved the final manuscript.

\section{Ethics approval and consent to participate}

All human tissue samples were collected by The Second Affiliated Hospital of Kunming Medical University and written informed consent was obtained from all patients. All methods were approved by the Research Medical Ethics Committee of Kunming Medical University and were performed in accordance with the approved guidelines.

\section{Patient consent for publication}

Not applicable.

\section{Competing interests}

The authors declare that they have no competing interests.

\section{References}

1. Siegel RL, Miller KD and Jemal A: Cancer statistics, 2015. CA Cancer J Clin 65: 5-29, 2015.

2. Penas-Prado M, Armstrong S and Gilbert MR: Glioblastoma. Handb Clin Neurol 105: 485-506, 2012.

3. Smith JS, Brachmann CB, Celic I, Kenna MA, Muhammad S, Starai VJ, Avalos JL, Escalante-Semerena JC, Grubmeyer C, Wolberger C and Boeke JD: A phylogenetically conserved NAD+-dependent protein deacetylase activity in the Sir 2 protein family. Proc Natl Acad Sci USA 97: 6658-6663, 2000.

4. Liu T, Liu PY and Marshall GM: The critical role of the class III histone deacetylase SIRT1 in cancer. Cancer Res 69: 1702-1705, 2009.

5. Lin SJ, Defossez PA and Guarente L: Requirement of NAD and SIR2 for life-span extension by calorie restriction in Saccharomyces cerevisiae. Science 289: 2126-2128, 2000.

6. Marumoto T and Saya H: Molecular biology of glioma. Adv Exp Med Biol 746: 2-11, 2012.

7. Luo J, Nikolaev AY, Imai S, Chen D, Su F, Shiloh A, Guarente L and $\mathrm{Gu}$ W: Negative control of p53 by Sir2alpha promotes cell survival under stress. Cell 107: 137-148, 2001.

8. Vaziri H, Dessain SK, Ng Eaton E, Imai SI, Frye RA, Pandita TK, Guarente L and Weinberg RA: hSIR2(SIRT1) functions as an NAD-dependent p53 deacetylase. Cell 107: 149-159, 2001.

9. Lim CS: Human SIRT1: A potential biomarker for tumorigenesis? Cell Biol Int 31: 636-637, 2007.

10. Choi HN, Bae JS, Jamiyandorj U, Noh SJ, Park HS, Jang KY, Chung MJ, Kang MJ, Lee DG and Moon WS: Expression and role of SIRT1 in hepatocellular carcinoma. Oncol Rep 26: 503-510, 2011.

11. Holloway KR, Calhoun TN, Saxena M, Metoyer CF, Kandler EF, Rivera CA and Pruitt K: SIRT1 regulates Dishevelled proteins and promotes transient and constitutive Wnt signaling. Proc Natl Acad Sci USA 107: 9216-9221, 2010. 
12. Zhang Y, Zhang M, Dong H, Yong S, Li X, Olashaw N, Kruk PA, Cheng JQ, Bai W, Chen J, et al: Deacetylation of cortactin by SIRT1 promotes cell migration. Oncogene 28: 445-460, 2009.

13. Firestein R, Blander G, Michan S, Oberdoerffer P, Ogino S, Campbell J, Bhimavarapu A, Luikenhuis S, de Cabo R Fuchs C, et al: The SIRT1 deacetylase suppresses intestinal tumorigenesis and colon cancer growth. PLoS One 3: e2020, 2008

14. Pruitt K, Zinn RL, Ohm JE, McGarvey KM, Kang SH, Watkins DN, Herman JG and Baylin SB: Inhibition of SIRT1 reactivates silenced cancer genes without loss of promoter DNA hypermethylation. PLoS Genet 2: e40, 2006.

15. Yeung F, Hoberg JE, Ramsey CS, Keller MD, Jones DR, Frye RA and Mayo MW: Modulation of NF-kappaB-dependent transcription and cell survival by the SIRT1 deacetylase. EMBO J 23: 2369-2380, 2004.

16. Chua KF, Mostoslavsky R, Lombard DB, Pang WW, Saito S, Franco S, Kaushal D, Cheng HL, Fischer MR, Stokes N, et al: Mammalian SIRT1 limits replicative life span in response to chronic genotoxic stress. Cell Metab 2: 67-76, 2005

17. Zavadil J, Haley J, Kalluri R, Muthuswamy SK and Thompson E: Epithelial-mesenchymal transition. Cancer Res 68: 9574-9577, 2008.

18. Kalluri R and Weinberg RA: The basics of epithelial-mesenchymal transition. J Clin Invest 119: 1420-1428, 2009.

19. Miyazono K: Transforming growth factor- $\beta$ signaling and cancer: The 28th sapporo cancer seminar, 25-27 June 2008. Cancer Sci 100: 363-365, 2009.

20. Cui Y, Li J, Zheng F, Ouyang Y, Chen X, Zhang L, Chen Y, Wang L, Mu S and Zhang H: Effect of SIRT1 Gene on epithelial-mesenchymal transition of human prostate cancer PC-3 Cells. Med Sci Monit 22: 380-386, 2016.

21. Cheng F, Su L, Yao C, Liu L, Shen J, Liu C, Chen X, Luo Y, Jiang L, Shan J, et al: SIRT1 promotes epithelial-mesenchymal transition and metastasis in colorectal cancer by regulating Fra-1 expression. Cancer Lett 375: 274-283, 2016.

22. Simic P, Williams EO, Bell EL, Gong JJ, Bonkowski M and Guarente L: SIRT1 suppresses the epithelial-to-mesenchymal transition in cancer metastasis and organ fibrosis. Cell Rep 3 : 1175-1186, 2013

23. Lee M, Kim DW, Yoon H, So D, Khalmuratova R, Rhee CS, Park JW and Shin HW: Sirtuin 1 attenuates nasal polypogenesis by suppressing epithelial-to-mesenchymal transition. J Allergy Clin Immunol 137: 87-98.e7, 2016.

24. Qu Y, Zhang J, Wu S, Li B, Liu S and Cheng J: SIRT1 promotes proliferation and inhibits apoptosis of human malignant glioma cell lines. Neurosci Lett 525: 168-172, 2012.

25. Lee JS, Park JR, Kwon OS, Lee TH, Nakano I, Miyoshi H, Chun KH, Park MJ, Lee HJ, Kim SU and Cha HJ: SIRT1 is required for oncogenic transformation of neural stem cells and for the survival of 'cancer cells with neural stemness' in a p53-dependent manner. Neuro Oncol 17: 95-106, 2015.

26. Wu WB, Wang W, Du YH, Li H, Xia SJ and Liu HT: MicroRNA-3713 regulates bladder cell invasion via MMP9. Sci Rep 6: 32374, 2016.

27. Sun T, Fu J, Shen T, Lin X, Liao L, Feng XH and Xu J: The Small C-terminal domain phosphatase 1 inhibits cancer cell migration and invasion by dephosphorylating $\operatorname{Ser}(\mathrm{P}) 68$-Twist1 to accelerate Twistl protein degradation. J Biol Chem 291: 11518-11528, 2016

28. Ford J, Jiang M and Milner J: Cancer-specific functions of SIRT1 enable human epithelial cancer cell growth and survival. Cancer Res 65: 10457-10463, 2005.

29. Liu Z, Long X, Chao C, Yan C, Wu Q, Hua S, Zhang Y, Wu A and Fang W: Knocking down CDK4 mediates the elevation of let-7c suppressing cell growth in nasopharyngeal carcinoma. BMC Cancer 14: 274, 2014.
30. Livak KJ and Schmittgen TD: Analysis of relative gene expression data using real-time quantitative PCR and the 2(-Delta Delta C(T)) method. Methods 25: 402-408, 2001.

31. Li J, Qu Q, Qu J, Luo WM, Wang SY, He YZ, Luo QS, Xu YX and Wang YF: Association between XRCC1 polymorphisms and glioma risk among Chinese population. Med Oncol 31: 186, 2014.

32. Sai K, Zhong MG, Wang J, Chen YS, Mou YG, Ke C, Zhang XH, Yang QY, Lin FH, Guo CC, et al: Safety evaluation of high-dose BCNU-loaded biodegradable implants in Chinese patients with recurrent malignant gliomas. J Neurol Sci 343: 60-65, 2014.

33. Zhou Q: WHO classification of tumors of central nervous system (2007): An introduction. Zhonghua Bing Li Xue Za Zhi 37: 5-7, 2008 (In Chinese).

34. Ohgaki H and Kleihues P: Epidemiology and etiology of gliomas. Acta Neuropathol 109: 93-108, 2005.

35. Bosch-Presegué L and Vaquero A: The dual role of sirtuins in cancer. Genes Cancer 2: 648-662, 2011.

36. Chen X, Hokka D, Maniwa Y, Ohbayashi C, Itoh T and Hayashi Y: Sirt1 is a tumor promoter in lung adenocarcinoma. Oncol Lett 8: 387-393, 2014.

37. Yu DF, Jiang SJ, Pan ZP, Cheng WD, Zhang WJ, Yao XK, Li YC and Lun YZ: Expression and clinical significance of Sirt1 in colorectal cancer. Oncol Lett 11: 1167-1172, 2016.

38. Oon CE, Strell C, Yeong KY, Östman A and Prakash J: SIRT1 inhibition in pancreatic cancer models: Contrasting effects in vitro and in vivo. Eur J Pharmacol 757: 59-67, 2015

39. Yuan F, Liu L, Lei Y and Tang P: p53 inhibits the upregulation of sirtuin 1 expression induced by c-Myc. Oncol Lett 14: 4396-4402, 2017

40. Voulgari A and Pintzas A: Epithelial-mesenchymal transition in cancer metastasis: Mechanisms, markers and strategies to overcome drug resistance in the clinic. Biochim Biophys Acta 1796: 75-90, 2009.

41. Thiery JP and Sleeman JP: Complex networks orchestrate epithelial-mesenchymal transitions. Nat Rev Mol Cell Biol 7: 131-142, 2006.

42. Kudo-Saito C, Shirako H, Takeuchi T and Kawakami Y: Cancer metastasis is accelerated through immunosuppression during Snail-induced EMT of cancer cells. Cancer Cell 15: 195-206, 2009.

43. Kang Y and Massagué J: Epithelial-mesenchymal transitions: Twist in development and metastasis. Cell 118: 277-279, 2004.

44. Yilmaz M and Christofori G: EMT, the cytoskeleton, and cancer cell invasion. Cancer Metastasis Rev 28: 15-33, 2009.

45. Liu ZJ, Liu HL, Zhou HC and Wang GC: TIPE2 Inhibits Hypoxia-Induced Wnt/ $\beta$-catenin pathway activation and EMT in glioma cells. Oncol Res 24: 255-261, 2016.

46. Wang Z, Wu Y, Wang Y, Jin Y, Ma X, Zhang Y and Ren $\mathrm{H}$ : Matrine inhibits the invasive properties of human glioma cells by regulating epithelial-to-mesenchymal transition. Mol Med Rep 11: 3682-3686, 2015

47. Byles V, Zhu L, Lovaas JD, Chmilewski LK, Wang J, Faller DV and Dai Y: SIRT1 induces EMT by cooperating with EMT transcription factors and enhances prostate cancer cell migration and metastasis. Oncogene 31: 4619-4629, 2012.

This work is licensed under a Creative Commons Attribution-NonCommercial-NoDerivatives 4.0 International (CC BY-NC-ND 4.0) License. 